\title{
Globus Pallidus Discharge Is Coincident with Striatal Activity during Global Slow Wave Activity in the Rat
}

\author{
Joshua A. Goldberg, ${ }^{1}$ Svetlana S. Kats, ${ }^{2}$ and Dieter Jaeger ${ }^{2}$ \\ ${ }^{1}$ Department of Physiology, The Hebrew University-Hadassah Medical School, and the Interdisciplinary Center for Neural Computation, The Hebrew \\ University, Jerusalem, 91120, Israel, and 2Department of Biology, Emory University, Atlanta, Georgia 30322
}

The emergence of bursting and oscillations in the basal ganglia under normal and pathological conditions has attracted considerable interest, but the neural substrate of these patterns is poorly understood. Here we use multisite recordings in anesthetized rats to examine the relationship of globus pallidus (GP) spiking and striatal activity in relation to cortical slow-wave activity. We found that GP neurons displayed increased spike rates or bursts coincident with cortical activation and striatal up states. Furthermore, the onset of GP bursts typically coincides with transitions to striatal up states that precede striatal spiking. These data indicate that GP activity is driven by excitatory corticosubthalamic input during periods of synchronized bursting activity.

Key words: basal ganglia; synchrony; bursting; subthalamic nucleus; Parkinson's disease; inhibition

\section{Introduction}

In the classical model of basal ganglia (BG) circuitry, the striatum is considered the sole structure transmitting cortical input (Albin et al., 1989). The major input to the globus pallidus (GP) was thus presumed to be inhibitory and of striatal origin. This view is supported by the fact that $>80 \%$ of the synapses on GP neurons are formed by striatal neurons (Shink and Smith, 1995). However, microstimulation studies demonstrated that the cortex also exerts a fast excitatory influence on GP activity via the corticosubthalamic nucleus (cortico-STN) pathway (Ryan and Clark, 1991; Kita, 1992; Nambu et al., 2000). The first goal of our study was to determine whether striatal input does indeed dominate the control of GP activity during cortical slow-wave activity (SWA).

In anesthesia-induced SWA, the neuronal activity within the cortical-BG circuitry exhibits $\sim 1 \mathrm{~Hz}$ oscillations (Magill et al., 2000; Mahon et al., 2001; Tseng et al., 2001). The global cortical synchronization of these oscillations enables the study of the relative timing of the neuronal activity among BG structures, thereby revealing the interplay between different BG pathways. Thus, cortical SWA is correlated with bursting of neurons in the GP and STN, albeit with variable phase relationships (Magill et al., 2000). In the striatum, the SWA produces synchronous down states and up states in medium spiny projection neurons (MSNs), and spiking occurs only in the up states, which coincides with upswings in the cortical EEG (Wilson and Groves, 1981; Wilson and Kawaguchi, 1996; Stern et al., 1998; Mahon et al., 2001; Tseng et al., 2001). Thus, our second goal was to determine the precise

\footnotetext{
Received May 26, 2003; revised Sept. 20, 2003; accepted Sept. 22, 2003.

This work was supported by Grant R01-NS39852 to D.J. by the National Institute of Neurological Disorders and Stroke and by the Michael J. Fox Foundation. J.A.G. was supported by a summer fellowship from the Parkinson's Disease Foundation and by the Yeshaya Horowitz Association. S.S.K. was supported by a summer undergraduate research program at Emory fellowship.

Correspondence should be addressed to Dieter Jaeger, Department of Biology, Emory University, 1510 Clifton Road, Atlanta, GA 30322. E-mail: djaeger@emory.edu.

Copyright $\odot 2003$ Society for Neuroscience $\quad$ 0270-6474/03/2310058-06\$15.00/0
}

phase relationship among the following during SWA: (1) cortical activity, (2) striatal up-down fluctuations, and (3) GP discharge. Understanding the flow of information in the BG during SWA may improve our understanding of the propagation of rhythmic activity in parkinsonian states (Bergman et al., 1994; Nini et al., 1995; Levy et al., 2000; Raz et al., 2000).

To address these goals, we simultaneously recorded: (1) intracellularly from striatal MSNs, (2) extracellularly from GP neurons, and (3) cortical surface EEG. We hypothesize that if the striatum provides the dominant input to GP neurons, GP spiking would be inhibited during striatal up states. As a result, we would expect to find GP neurons firing out of phase with striatal up states during SWA. A third goal was to examine whether pallidal oscillations were strictly coupled to cortical inputs, or whether we could find any evidence for bursting activity independent of cortical input such as that which could be generated by an STN-GP feedback loop observed in organotypic cultures (Plenz and Kitai, 1999).

\section{Materials and Methods}

Electrophysiology. In vivo recordings were obtained from anesthetized rats $(n=17)$. All use of rats complied with National Institutes of Health guidelines on the care and use of laboratory animals and was approved by the Emory Institutional Animal Care and Use Committee. Male Sprague Dawley rats (50-100 d of age) were anesthetized with ketamine (75 mg/ $\mathrm{kg}$, i.p.), acepromazine $(0.75 \mathrm{mg} / \mathrm{kg}$, i.p.), and xylazine $(3.9 \mathrm{mg} / \mathrm{kg}$, i.p.) Supplementary doses of one-third of this amount of anesthetic were given when light paw-withdrawal reflexes developed, usually occurring at intervals of $1 \mathrm{hr}$. In some experiments, the supplementary dose was injected continuously using a syringe pump. The body temperature was maintained at $36^{\circ} \mathrm{C}$, and the heart rate was monitored. The cortex was exposed unilaterally to allow insertion of two glass microelectrodes, one for extracellular recording in GP, and the other for intracellular recording in striatum. GP recording tracks were aimed vertically at stereotaxic coordinates as follows: anteroposterior (AP), -1 to $-2 \mathrm{~mm}$ from bregma; lateral, 2.5-3.5 mm. After a GP recording, pontamine sky blue was ejected from the electrode with positive current (5-10 $\mu \mathrm{A}$ for 5-10 min). 
Striatal tracks were aimed for recording sites in the center of the dorsal striatum. Intracellular striatal recordings from MSNs were confirmed by a strong inward rectification in response to negative current injection and up-state-down-state cycling. Two skull screws were fastened either contralaterally ( 0 and $3 \mathrm{~mm}$ AP from bregma; $3 \mathrm{~mm}$ lateral) or one over each hemisphere above the sensorimotor cortex as electrodes to monitor EEG activity in reference to a subcutaneous ground wire.

Histology. After experiments, an injection of $0.5 \mathrm{ml}$ pentobarbital (50 $\mathrm{mg} / \mathrm{ml}$ ) was given, and rats were perfused transcardially with $10-15 \%$ buffered formalin (Sigma, St. Louis, MO ). Fixed brains were sectioned, and the locations of the GP recordings were verified histologically by locating the deposit of blue dye in 7 of the 12 animals from which GP neurons were recorded. In the remaining experiments, the GP neurons were identified by their depth, high firing rates, and narrow action potentials.

Data acquisition and analysis. Intracellular recordings were amplified $10 \times$ using a Neurodata IR283 amplifier (Cygnus, Branford, CT). Extracellular signals were amplified 10,000 $\times$ and bandpass filtered between 0.3 and $3 \mathrm{kHz}$ (model 1700 amplifier; A-M Systems, Carlsborg, WA), and EEG signals were amplified $1000 \times$ and bandpass filtered between 1 and $200 \mathrm{~Hz}$ (Bak Electronics, Mount Airy, MD). All data were digitized at 10 $\mathrm{kHz}$, and traces of $10 \mathrm{sec}$ duration were saved on a personal computer. Custom-made software was used to: (1) extract spike waveforms and spike times from the extracellular and intracellular recordings and (2) generate a version of the intracellular potential in which spikes were replaced by a straight-line segment. All subsequent data analysis was conducted using Matlab (MathWorks, Natick, MA). The analog data were downsampled to $400 \mathrm{~Hz}$, applying an anti-aliasing (low-pass) finite impulse response filter, and spike times were converted to a corresponding $2.5 \mathrm{msec}$ resolution.

Spectra were estimated by Welch's method (Percival and Walden, 1993). The threshold of the MSNs was determined as the point preceding a spike attaining a slope of $4 \mathrm{mV} / \mathrm{msec}$ (Wickens and Wilson, 1998; Tseng et al., 2001). Up and down transitions of MSNs were extracted from the subthreshold potential using a variant of the method proposed by Stern et al. (1998). The probability density function (pdf) $P_{\mathrm{V}}$ of the subthreshold potential $V$ was calculated from the all-points histogram of this signal (see Fig. 2b,f, top). A mixture-of-two-Gaussians model was fit to this pdf (solid line) using a constrained nonlinear least-square method (Levenberg-Marquardt algorithm). The two inflection points between the two peaks of the pdf were determined numerically (arrows). The subthreshold potential was low-pass filtered using a third-order Butterworth filter with a $4 \mathrm{~Hz}$ cutoff. Up (down) transitions were defined as the times when the filtered version of the potential crossed the depolarized (hyperpolarized) inflection point with a positive (negative) slope. Times of burst onset and offset in GP spike trains were determined using a published algorithm (Legéndy and Salcman, 1985) that searches for sequences of consecutive short interspike intervals (ISIs) that maximize a measure called the Poisson surprise (S). We excluded burst sequences identified by the algorithm that: (1) were composed of only two ISIs, (2) were $>1$ sec, or $(3)$ displayed only a small surprise measure $(S<2)$.

Confidence intervals (CIs) for the correlation functions involving an analog signal [cross-correlation functions and spike-triggered averages (STAs) ] were estimated by shuffling of the original data (time reversal of one of the signals or triggering on random events). CIs for the crossintensities of two point processes (spikes, up-down transitions and burst onsets-offsets) were estimated from the mean number of coincidences during a baseline period (at lags of $>1.9 \mathrm{sec}$ from time 0 ).

\section{Results}

\section{EEG and MSN during slow-wave activity}

The subthreshold potential of recorded MSNs fluctuated between depolarized up states and hyperpolarized down states (Fig. $1 a)$. The potential of the up states often remained below spike threshold, so that MSNs did not necessarily spike during every up state. The mean spike amplitude measured from the threshold was $61.9 \pm 1.9 \mathrm{mV}$ (mean $\pm \mathrm{SEM} ; n=24$ neurons). The power spectrum of the subthreshold voltage fluctuations exhibited a

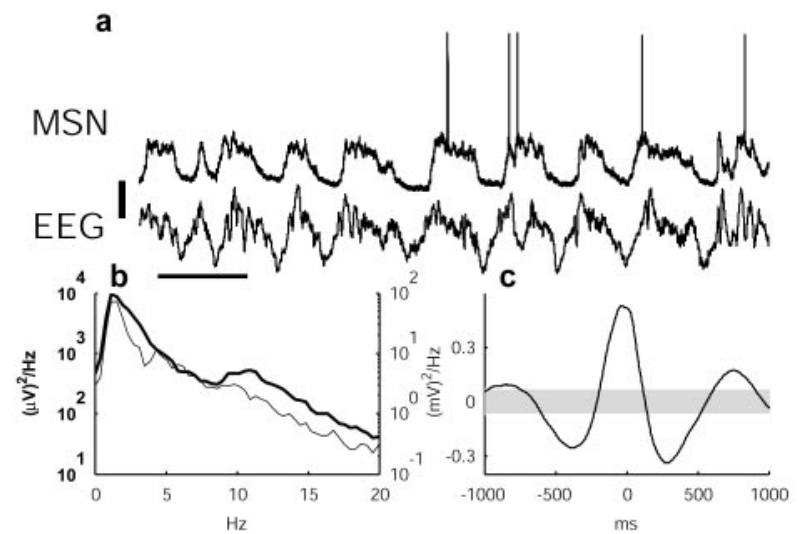

Figure 1. Synchronization of surface EEG with the subthreshold potential of a striatal MSN. a, Simultaneous MSN and surface EEG recording. Calibration: $1 \mathrm{sec}, 25 \mathrm{mV}$ in the MSN trace; 1 sec, $500 \mu \mathrm{V}$ in the EEG trace. $b$, Power spectra of the EEG (thick line, left scale) and the MSN potential fluctuations (thin line, right scale). c, Cross-correlation function (normalized to a correlation coefficient) between EEG and the subthreshold voltage of the MSN. Cls are indicated by the gray background.

peak at $1.3 \mathrm{~Hz}$, which attests to the periodicity of these fluctuations (Fig. 1b, thin trace). In agreement with previous studies (Mahon et al., 2001; Tseng et al., 2001), MSN up states were aligned with positive EEG deflections (Fig. 1a). The similarity of the rhythms of the two signals is demonstrated by a similar $1.3 \mathrm{~Hz}$ peak in the spectrum of the EEG (Fig. $1 b$, thick trace). Furthermore, the cross-correlation function calculated between these signals exhibited a highly significant peak (Fig. 1c) found in all MSN-EEG pairs simultaneously recorded. The population mean value of the maximal correlation coefficient between these signals was $0.45 \pm 0.03$. The mean latency of the peak was $1.3 \pm 6.5 \mathrm{msec}$, indicating that the EEG was in phase with MSN potential fluctuations. The EEG signals recorded from two electrodes at least 3 $\mathrm{mm}$ apart were virtually indistinguishable. These signals exhibited near perfect coherence $(0.6-1)$ with no phase lag in the frequency range of the SWA (data not shown), indicating that the SWA is truly a global cortical signal. The fact that the voltage fluctuations within any MSN are significantly correlated with the global EEG indicates that these fluctuations are a measure of the global SWA occurring in the cortex. Furthermore, because positive deflections in surface EEG of ketamine-xylazineanesthetized animals are correlated with neuronal discharge in the cortex (Contreras and Steriade, 1995) and with up-state transitions in corticostriatal neurons (Mahon et al., 2001), the strong relationship between a positive EEG deflection and a depolarization in the MSNs supports findings that striatal up states reflect cortical input (Wilson and Kawaguchi, 1996).

\section{Analysis of correlations in triple recordings}

Because all MSNs recorded during SWA were highly correlated with the global oscillations, we investigated how GP fits into this activity pattern by performing triple GP, MSN, and surface EEG recordings (Fig. 2). The mean firing rate of all GP neurons recorded was $20.3 \pm 1.9$ spikes per second $(n=29)$. Some GP neurons displayed continuous activity with some visible rate modulation (Fig. 2a), whereas others showed pauses during striatal down states (Fig. 2e). To quantify this relationship between GP spiking and the MSN potential $\left(V_{\mathrm{MSN}}\right)$, we calculated the firing rate of each GP neuron as a function of $V_{\mathrm{MSN}}$. This was achieved by first calculating the all-points histogram of $V_{\mathrm{MSN}}$ (normalized to a pdf $P_{\mathrm{V}}$ in Fig. $2 b, f$ ), which was generally bimodal 

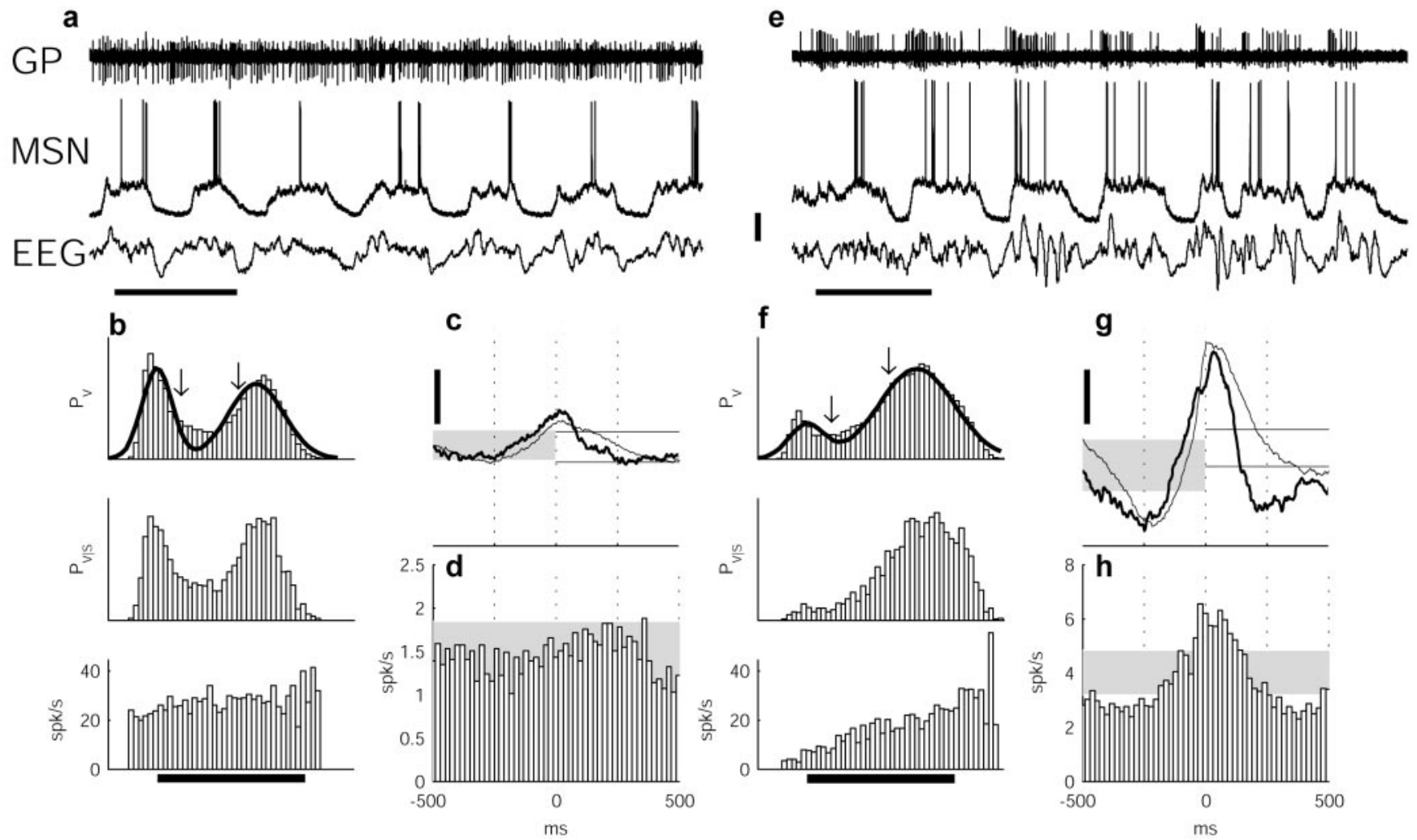

Figure 2. Global ketamine-induced oscillations in simultaneous cortical, striatal, and pallidal recordings. $a, e$, Two examples of simultaneous extracellular recordings of a GP neuron, an intracellular MSN recording, and surface EEG. Calibration: $1 \mathrm{sec}, 25 \mathrm{mV}$ in the MSN trace; $1 \mathrm{sec}, 500 \mu V$ in the EEG trace. $b$, $f$, The top panel shows the pdf $P_{V}$ of the subthreshold voltage $(V)$ of the $M S N \mathrm{~s}$ displayed in $a$ and $e$, respectively; the middle panel shows the $\operatorname{pdf} P_{\mathrm{v} / S}$ of the subthreshold voltage in the MSN at times of the emission of a spike (S) in the GP neuron; the bottom panel displays the firing rate of the GP neuron given the voltage in the MSN at the same time. Arrows denote inflection points between the two peaks of the pdf. Calibration, $30 \mathrm{mV}$. $c, g$, The thin line indicates the STA of the MSN potential about the emission (at time 0 ) of a spike in the GP neuron. (Is for the STA of MSNs are indicated by horizontal lines; the thick line shows the STA of the EEG at about the time of the emission of a spike in the GP neuron. CIs are indicated by the gray background. Calibration: $2 \mathrm{mV}$ in the averaged MSN trace; $27 \mu V$ in the averaged EEG trace. $d, h$, Conditional firing rate of the MSN about the emission of a spike in the GP neuron. Cls are indicated by the gray background. Dotted vertical lines in $c, d, g$, and $h$ show alignment of graphs at $-250,0$, and $+250 \mathrm{msec}$, respectively. spk/s, Spikes per second.

because of up and down states of MSN neurons. Then we calculated the histogram of $V_{\text {MSN }}$ at times corresponding with an emission of a spike by the GP neuron (Fig. $2 b, f, P_{\mathrm{V} / \mathrm{S}}$ ). Dividing the spike-times histogram by the all-points histogram and then by the sampling rate of $V_{\mathrm{MSN}}$ resulted in the required conditional GP firing rate. The examples shown demonstrate a GP neuron that displayed an insignificant correlation with $V_{\mathrm{MSN}}$ (Fig. 2a,b) and one with a strong tendency to only fire when the MSN was in the up state (Fig. 2e,f). A regression analysis of the dependence of the GP firing rate on the MSN potential revealed that in 23\% (3 of 13) of simultaneous GP and MSN recordings, the GP firing rate was independent of $V_{\mathrm{MSN}}$ (regression slope is not significantly different from zero), whereas in 77\% (10 of 13) of cases, the GP firing rate increased significantly with MSN depolarization.

STA can show whether GP spikes are related to fluctuations in $V_{\mathrm{MSN}}$ with a precise temporal alignment. The waveform of the STAs typically displayed a strong upswing around time 0 (Fig. $2 g$, thin line) that was still present in a weaker form for GP neurons without distinct pauses (Fig. $2 c$, thin line). The STA waveform was significantly different from flat for all GP-MSN pairs recorded, which was quantified by the ratio of the range of the STA waveform (trough to peak) to the CI of the estimate of the STA (Fig. 2c,g, distance between horizontal lines). The mean value of this significance index was $3.95 \pm 0.45(n=14)$. Additionally, in $79 \%$ ( 11 of 14 ) of the pairs, there was a significant increase in the MSN firing rate around the emission of GP spikes (Fig. $2 h$ ). Be- cause $V_{\text {MSN }}$ was highly correlated with the EEG (Fig. 1) as well as with GP spiking, we expected that GP spiking would be correlated with the EEG as well. In fact, the STA of the EEG on GP spikes (Fig. 2c,g, thick line) shared remarkably similar characteristics with the STA of the MSN potential. The mean value of the corresponding significance index for this signal was $3.1 \pm 0.2(n=29)$. The STA waveforms can be used to evaluate the timing of GP spiking relative to the global oscillation expressed in the EEG and MSNs. The population mean of the latency of the peak in the STA of the MSN potential was $67 \pm 22 \mathrm{msec}$. The corresponding mean latency estimated from the EEG was $49 \pm 21 \mathrm{msec}$. Both estimates indicate that GP spikes tend to occur during upswings in cortical and MSN activity and that they precede maximal depolarization by $\sim 50 \mathrm{msec}$ on average. This finding indicates that both MSN and GP activity were tightly linked to EEG slow-wave activity. However, because the spikes from all phases in the SWA cycle were used to construct STA waveforms, a better method to examine phase relationships between structures is to align the activity between structures on specific transition points in the SWA cycle. Below, we performed such analyses for transition points in MSN subthreshold potentials and GP spike rate.

\section{MSN state transitions}

The up-state and down-state transition points of MSN neurons were determined (see Materials and Methods). The population mean durations of the up and down states were $427 \pm 43$ and 

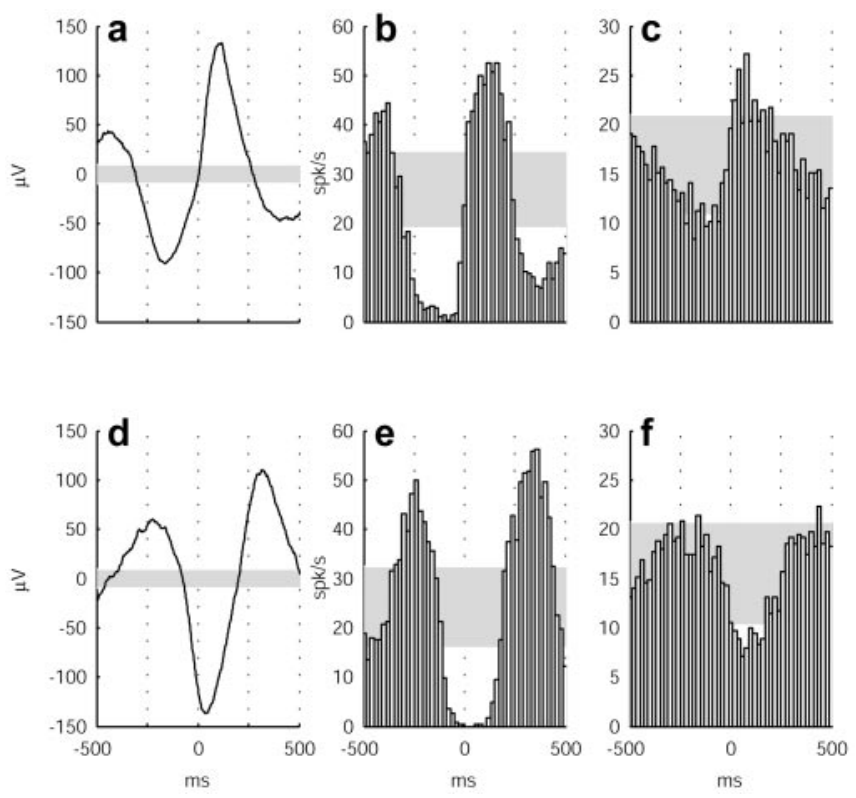

Figure 3. Relationship of EEG and GP discharge to up and down transitions in the striata MSN. $a$, Population mean of all averaged EEG waveforms triggered on up transitions. $b$, $c$, Two examples of the conditional firing rate of a GP neuron about the time of up transitions. $d$, Population mean of all averaged EEG waveforms triggered on down transitions. e, f, Two examples of the conditional firing rate of the same GP neurons (displayed in $b$ and c, respectively) about the time of down transitions. Cls are indicated by the gray background. Dotted vertical lines show 250 msec increments along the $x$-axis. spk/s, Spikes per second.

$233 \pm 17 \mathrm{msec}$, respectively ( $n=20$ neurons). This is in good agreement with a previous study, which also found a similar pattern for corticostriatal neurons (Stern et al., 1997). The mean firing rate of the MSNs during the up state was $8.4 \pm 1.7$ spikes per second, which is higher than what we observed with extracellular recordings in the same preparation (data not shown), probably because intracellular recording causes a depolarizing shunt. We used MSN state transition points as event markers to examine the correlation between the three recorded structures at transition times of MSN neurons. All EEG waveforms triggered on MSN up transitions were significant and highly reproducible across MSN-EEG pairs (Fig. $3 a$ ), indicating that up transitions were coupled with a large and consistent upswing in the EEG signal. The population mean latency (across MSN-EEG pairs) from the trough of the EEG waveform to the up transition was $166 \pm 11 \mathrm{msec}(n=17)$. The spike activity of GP neurons typically displayed a dramatic increase in firing coincident with MSN up-state transitions, whereas the firing rate was much below the mean rate before the up transition (Fig. $3 b$ ). The effect was significant in $85 \%$ (11 of 13 ) of the GP-MSN pairs, although its strength varied among pairs (Fig. $3 b, c$ ). The population mean latency (across pairs that exhibited a significant trough) from the trough of the conditional firing rate to the up transition was $160 \pm 32 \mathrm{msec}(n=10)$

Performing the same analysis with regard to the down transition demonstrated that the EEG and the GP firing rates were modulated just as strongly around the end of striatal up states (Fig. $3 d-f$ ). The EEG waveforms triggered on down transitions were significant for all recordings and highly reproducible in terms of the location of the peaks and troughs nearest to the transition time. The population mean latency (across MSN-EEG pairs) from the down transition to the trough in the EEG waveforms was $46 \pm 8 \mathrm{msec}(n=20)$. This correlation was mirrored
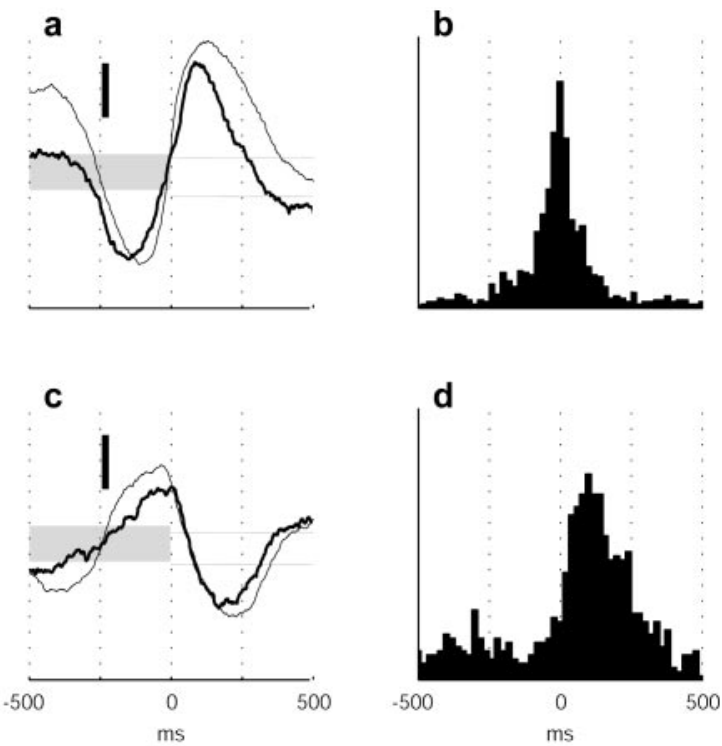

Figure 4. The relationship of EEG, MSN potential, and up or down transitions to the onset and offset of GP bursts. $a$, Population mean of all significant averaged waveforms of the EEG (thick line; $\mathrm{Cl}$ is indicated by a gray background) and MSN potential (thin line; $\mathrm{Cl}$ is indicated by horizontal thin gray lines) triggered on the onset of GP bursts. $b$, Histogram of the latency from burst onsets to the nearest up transition (all neuronal pairs pooled). c, Population mean of all significant averaged waveforms of the EEG and MSN potential triggered on the offset of a GP burst. $d$, Histogram of the latency from GP burst offsets to nearest MSN down transitions. Dotted vertical lines show 250 msec increments along the $x$-axis. Calibration: $3 \mathrm{mV}$ in the averaged MSN trace; $40 \mu \mathrm{V}$ in the averaged EEG trace.

by a concomitant change in GP firing rate (Fig. $3 e, f$ ), which was significant in 69\% (9 of 13) of MSN-GP pairs recorded. The population mean latency from the down transition to the trough of the conditional firing rate was $54 \pm 14 \mathrm{msec}$.

\section{GP bursting}

The abrupt changes in the GP firing rate around the time of MSN up and down transitions suggests that the occurrence of GP spike bursting is related to the same process that generates MSN state transitions. To examine this question for all GP bursts regardless of their relationship with MSN or EEG potentials, we implemented an automated burst detection algorithm (see Materials and Methods). We then used the detected burst on and offset times as event markers to test how they were related to MSN and EEG potentials. The average waveforms generated by triggering the EEG on the GP burst onsets were more variable than those generated by triggering on the MSN up transition. Nevertheless, $66 \%$ (19 of 29) of paired recordings showed a significant EEG trough before GP burst onset followed by an EEG peak (Fig. $4 a$, thick line). The population mean latency (across significant GPEEG pairs) from the EEG trough to the GP burst onset was $150 \pm$ $17 \mathrm{msec}$. Averaging the MSN potential on the GP burst onset proved to be even more robust in that $85 \%$ (11 of 13) of recorded pairs were significant and generated a waveform very similar to the averaged EEG waveform (Fig. $4 a$, thin line). The graphs indicate that GP burst onset, EEG depolarization, and MSN depolarization are highly coupled and precisely in phase. The tight linkage with MSN up transitions is verified by calculating the distribution of the latency from a GP burst onset to the nearest up transition (Fig. 4b). Indeed, this distribution was centered on time 0 , indicating that the majority of GP burst onsets coincided with MSN up transitions.

Triggering of the EEG and the MSN potential on the offset of 
the GP bursts resulted in significant waveforms in 62\% (18 of 29) of the GP-EEG pairs, and likewise 62\% (8 of 13) of the GP-MSN pairs. These triggered EEG and MSN waveforms were very similar to each other (Fig. $4 c$ ) and demonstrate that the offset of a burst marks a potential drop in both signals. The burst offset coincided with the end of EEG and MSN depolarization and preceded a subsequent trough by $213 \pm 23 \mathrm{msec}$ in the EEG and $234 \pm 36 \mathrm{msec}$ in the MSN potential. The distribution of the latency from a GP burst offset to the nearest MSN down transition (Fig. $4 d$ ) indicates that GP burst offsets typically precede MSN down transitions by $100-150 \mathrm{msec}$. This was approximately the same delay that it took for an MSN neuron to reach the down-state transition point from a plateau depolarization.

\section{Discussion}

In agreement with previous studies (Magill et al., 2000), we found that under ketamine-xylazine anesthesia, the corticobasal ganglia circuit participates in global SWA with a frequency of $\sim 1 \mathrm{~Hz}$ (Magill et al., 2000; Mahon et al., 2001; Tseng et al., 2001). In addition, we found that positive surface EEG deflections and MSN up states were precisely in phase. Because positive deflections in the EEG represent increased neuronal discharge in the cortex (Contreras and Steriade, 1995), our results support previous findings that cortical activity is required for the maintenance of MSN up states (Wilson, 1993). In fact, corticostriatal neurons show up-state transitions with positive EEG deflections (Mahon et al., 2001) and up-state distributions very similar to MSNs (Stern et al., 1997), suggesting that the observed onset and offset alignment of MSN up states with surface EEG directly relates to tightly coupled global up states in both the cortex and striatum.

A globally synchronized up state among striatal MSNs (Stern et al., 1998) leads to the prediction that GP should be depressed strongly at these times because of the heavy GABAergic striatal projection onto GP neurons (Shink and Smith, 1995). In fact, we found that GP neurons fired more vigorously when the potential of the MSNs was increasingly more depolarized. Furthermore, the onset and the offset of GP bursts coincided with up and down transitions in the MSN potential, respectively. Thus, in the state of ketamine-induced SWA, cortical and BG circuits were tightly coupled, and there was no evidence for MSN up states or GP bursting independent of cortical activity. The latter result suggests that the STN and GP were not in a mode of feedback oscillations that has been observed in organotypic cultures (Plenz and Kitai, 1999).

Because the MSN state transitions mirrored changes in cortical activity, it seems likely that the onset of GP bursts is caused by cortical activity by way of a pathway that circumvents the striatum. Cortical microstimulation studies have shown an early latency excitation in GP neurons followed by inhibition (Ryan and Clark, 1991; Kita, 1992; Nambu et al., 2000). The early component was attributed to the corticosubthalamic pathway and the inhibition to the striatal pathway, suggesting that cortical input reaches the GP first via the STN and only later via the striatum. Thus, our data are most consistent with an entrainment of GP activity by excitatory STN input. The fast effect of cortico-STN excitation explains why GP firing rates increased as soon as cortical volleys led to MSN up-state transitions. Because MSNs usually spike well after the up-state transition point occurs (as defined in our algorithm), it is unlikely that striatal inputs to the GP were responsible for observed GP spike-rate increases even if the GABAergic striatal input were somehow excitatory. Nevertheless, once the up state of the SWA cycle is initiated, it is possible that the striatal input affects precise spike timing in the GP by increas- ing postsynaptic inhibitory conductances (Häusser and Clark, 1997) or the degree of burstiness exhibited by individual neurons by activating low-threshold calcium currents (Cooper and Stanford, 2000). Interestingly, increases in GP discharge that precede the MSN up state may even contribute to their generation by disinhibiting the GABAergic striatal interneurons (Bolam et al., 2000). A previous study found some GP neurons that were antiphasic with respect to surface EEG and with another simultaneously recorded GP neuron (Magill et al., 2000). We did not encounter such an antiphasic relationship among our sample of 29 recorded GP neurons. The difference could be attributable to a different region of the GP being sampled or possibly a different sampling bias among cell types in the GP. We can certainly not exclude the possibility that for some GP neurons, the balance of striatal inhibitory and STN excitatory input is tilted toward striatal dominance.

The network connections by which the different basal ganglia nuclei participate in the observed SWA activity may be relevant to other rhythms that are found in the corticobasal ganglia circuitry, such as during parkinsonian activity (Bergman et al., 1994; Hutchison et al., 1997; Raz et al., 2000). The overriding influence of the cortico-STN pathway on pallidal activity during anesthesia-induced SWA observed in the present study contributes to our understanding of why the STN is generally the preferred location for deep-brain stimulation when treating parkinsonian symptoms (Hashimoto et al., 2003).

\section{References}

Albin RL, Young AB, Penney JB (1989) The functional anatomy of basal ganglia disorders. Trends Neurosci 12:366-375.

Bergman H, Wichmann T, Karmon B, DeLong MR (1994) The primate subthalamic nucleus. II. Neuronal activity in the MPTP model of Parkinsonism. J Neurophysiol 72:507-520.

Bolam JP, Hanley JJ, Booth PA, Bevan MD (2000) Synaptic organisation of the basal ganglia. J Anat 196:527-542.

Contreras D, Steriade M (1995) Cellular basis of EEG slow rhythms: a study of dynamic corticothalamic relationships. J Neurosci 15:604-622.

Cooper AJ, Stanford IM (2000) Electrophysiological and morphological characteristics of three subtypes of rat globus pallidus neurone in vitro. J Physiol (Lond) 527:291-304.

Hashimoto T, Elder CM, Okun MS, Patrick SK, Vitek JL (2003) Stimulation of the subthalamic nucleus changes the firing pattern of pallidal neurons. J Neurosci 23:1916-1923.

Häusser M, Clark BA (1997) Tonic synaptic inhibition modulates neuronal output pattern and spatiotemporal synaptic integration. Neuron 19:665-678.

Hutchison WD, Lozano AM, Tasker RR, Lang AE, Dostrovsky JO (1997) Identification and characterization of neurons with tremor-frequency activity in human globus pallidus. Exp Brain Res 113:557-563.

Kita H (1992) Responses of globus pallidus neurons to cortical stimulation: intracellular study in the rat. Brain Res 589:84-90.

Legéndy CR, Salcman M (1985) Bursts and recurrences of bursts in the spike trains of spontaneously active striate cortex neurons. J Neurophysiol 53:926-939.

Levy R, Hutchison WD, Lozano AM, Dostrovsky JO (2000) High-frequency synchronization of neural activity in the subthalamic nucleus of Parkinsonian patients with limb tremor. J Neurosci 20:7766-7775.

Magill PJ, Bolam JP, Bevan MD (2000) Relationship of activity in the subthalamic nucleus-globus pallidus network to cortical electroencephalogram. J Neurosci 20:820-833.

Mahon S, Deniau JM, Charpier S (2001) Relationship between EEG potentials and intracellular activity of striatal and cortico-striatal neurons: an in vivo study under different anesthetics. Cereb Cortex 11:360-373.

Nambu A, Tokuno H, Hamada I, Kita H, Imanishi M, Akazawa T, Ikeuchi Y, Hasegawa N (2000) Excitatory cortical inputs to pallidal neurons via the subthalamic nucleus in the monkey. J Neurophysiol 84:289-300.

Nini A, Feingold A, Slovin H, Bergman H (1995) Neurons in the globus pallidus do not show correlated activity in the normal monkey, but phase- 
locked oscillations appear in the MPTP model of Parkinsonism. J Neurophysiol 74:1800-1805.

Percival D, Walden A (1993) Spectral analysis for physical applications: multitaper and conventional univariate techniques. Cambridge, UK: Cambridge UP.

Plenz D, Kitai ST (1999) A basal ganglia pacemaker formed by the subthalamic nucleus and external globus pallidus. Nature 400:677-682.

Raz A, Vaadia E, Bergman H (2000) Firing patterns and correlations of spontaneous discharge of pallidal neurons in the normal and the tremulous 1-methyl-4-phenyl-1,2,3,6-tetrahydropyridine vervet model of Parkinsonism. J Neurosci 20:8559-8571.

Ryan LJ, Clark KB (1991) The role of the subthalamic nucleus in the response of globus pallidus neurons to stimulation of the prelimbic and agranular frontal cortices in rats. Exp Brain Res 86:641-651.

Shink E, Smith Y (1995) Differential synaptic innervation of neurons in the internal and external segments of the globus pallidus by the GABA- and glutamate-containing terminal in squirrel monkey. J Comp Neurol 358:119-141.

Stern EA, Kincaid AE, Wilson CJ (1997) Spontaneous subthreshold mem- brane potential fluctuations and action potential variability of rat corticostriatal and striatal neurons in vivo. J Neurophysiol 77:1697-1715.

Stern EA, Jaeger D, Wilson CJ (1998) Membrane potential synchrony of simultaneously recorded striatal spiny neurons in vivo. Nature 394:475-478.

Tseng KY, Kasanetz F, Kargieman L, Riquelme LA, Murer MG (2001) Cortical slow oscillatory activity is reflected in the membrane potential and spike trains of striatal neurons in rats with chronic nigrostriatal lesions. J Neurosci 21:6430-6439.

Wickens JR, Wilson CJ (1998) Regulation of action-potential firing in spiny neurons of the rat neostriatum in vivo. J Neurophysiol 79:2358-2364.

Wilson CJ (1993) The generation of natural firing patterns in neostriatal neurons. In: Progress in brain research, Vol 99 (Arbuthnott GW, Emson PC, eds), pp 277-296. Amsterdam: Elsevier.

Wilson CJ, Groves PM (1981) Spontaneous firing patterns of identified spiny neurons in the rat neostriatum. Brain Res 220:67-80.

Wilson CJ, Kawaguchi Y (1996) The origins of two-state spontaneous membrane potential fluctuations of neostriatal spiny neurons. J Neurosci 16: 2397-2410. 